\title{
The high hall ventilation with the simplified simulation of the fan
}

\author{
Martin Kyncl $^{1, a}$ and Jaroslav Pelant ${ }^{1}$ \\ ${ }^{1}$ VZLÚ - Czech Aerospace Research Centre, Beranových 130, 19905 Praha - Letňany, Czech Republic
}

\begin{abstract}
Here we work with the system of equations describing the non-stationary compressible turbulent multi-component flow in the gravitational field. We focus on the numerical simulation of the fan situated inside the high hall. The RANS equations are discretized with the use of the finite volume method. The original modification of the Riemann problem and its solution is used at the boundaries. The combination of specific boundary conditions is used for the simulation of the fan. The presented computational results are computed with own-developed code (C, FORTRAN, multiprocessor, unstructured meshes in general).
\end{abstract}

\section{Introduction}

In this contribution we consider the Reynolds-Averaged Navier-Stokes equations (RANS) with the k-omega model of turbulence, shown in [1,2]. We focus on the real gas flow in the gravitational field. The special method for the simulation of the simple fan is presented, using two compatible boundary conditions, used previously in [3]. We focus on the numerical solution of these equations, using owndeveloped software.

\section{Methods}

For the discretization of the system we use either explicit or implicit finite volume method in order to discretize the analytical problem, represented by the system of equations in generalized (integral) form. In order to apply this method we split the area of the interest into the elements, and we construct a piecewise constant solution in time, as described in [4]. The crucial problem of this method lies in the evaluation of the so-called fluxes (and its Jacobians) through the edges/faces of the particular elements. We use the exact Riemann solver for the solution of the local problem at each face, with the theory shown in [5]. At the boundary faces it is necessary to solve the incomplete Riemann problem, where the right-hand side initial condition is not known. It can be shown, that this right-hand side initial condition for the local problem can be partially replaced by the suitable complementary condition. Various original modifications of the Riemann problem (and exact solutions of these modifications) were shown and analyzed in [6-10]. These boundary conditions were also used in [11, $14,13]$. Here we present the simulation of the fan, using two connected boundaries. At these boundaries we solve the conservation laws, using the modification of the Riemann problem by the preference of the total quantities at the inlet, and the modification by the preference of the mass flow at the outlet. Using such boundary conditions it

\footnotetext{
a e-mail: kyncl@vzlu.cz
}

is necessary to compute the solution of the resulting nonlinear problems. The combination of these two boundary conditions was used previously for the simulation of the propeller disk in [3]. The partial boundary condition with the preference of mass flow is sometimes being implemented with the use of some iterative process, guessing the correct values (for the pressure, density, velocity) in order to match the given mass flow through the boundary. In our approach we try to be as exact as possible, using our own original procedures. We follow the exact solution of the initial-value problem for the system of hyperbolic partial differential equations, the original analysis was shown in [14]. The own-developed software (C, FORTRAN) is based on the finite volume method with the implicit or explicit time discretization, solution is computed on unstructured 3D meshes in general. Multiple types of parallelization are used OpenMPI (for the computers with shared memory), MPICH (for the computers with the distributed memory), CUDA (graphic cards computations) are used, see also [15-18]. The large linear systems within the implicit method are solved with the implemented preconditioned generalized minimal residual (GMRES) method.

\section{The Riemann problem for the split Euler equations}

For many numerical methods dealing with the two or three dimensional equations, describing the compressible flow, it is useful to solve the Riemann problem for the $3 D$ split Euler equations. We search the solution of the system of partial differential equations in time $t$ and space $(x, y, z)$

$$
\begin{aligned}
\frac{\partial \varrho}{\partial t}+\frac{\partial \varrho u}{\partial x} & =0 \\
\frac{\partial \varrho u}{\partial t}+\frac{\partial\left(p+\varrho u^{2}\right)}{\partial x} & =0 \\
\frac{\partial \varrho v}{\partial t}+\frac{\partial \varrho u v}{\partial x} & =0
\end{aligned}
$$




$$
\begin{aligned}
\frac{\partial \varrho w}{\partial t}+\frac{\partial \varrho u w}{\partial x} & =0 \\
\frac{\partial E}{\partial t}+\frac{\partial u(E+p)}{\partial x} & =0
\end{aligned}
$$

equipped with the initial conditions

$$
\begin{aligned}
& \varrho(x, t)=\varrho_{L}, \boldsymbol{v}(x, t)=\boldsymbol{v}_{L}, p(x, t)=p_{L}, \quad x<0, \\
& \varrho(x, t)=\varrho_{R}, \boldsymbol{v}(x, t)=\boldsymbol{v}_{R}, p(x, t)=p_{R}, \quad x>0 .
\end{aligned}
$$

Vector $\boldsymbol{v}=(u, v, w)$ denotes the velocity, $\varrho$ density, $p$ pressure, $E=\varrho e+\varrho|v|^{2}$ is the total energy, with $e$ denoting the specific internal energy. We assume the equation of state for the calorically ideal gas

$$
e=\frac{p}{\varrho(\gamma-1)}
$$

'Split' means here that we still have 5 equations in 3D, but the dependence on $y, z$ (space coordinates) is neglected, and we deal with the system for one space variable $x$. The system (1)-(5) is considered in the set $Q_{\infty}=(-\infty, \infty) \times$ $(0,+\infty)$

The solution of this problem is fundamentally the same as the solution of the Riemann problem for the 1D Euler equations, see [4, page 138]. In fact, the solution for the pressure, the first component of the velocity, and the density is exactly the same as in one-dimensional case. It is a characteristic feature of the hyperbolic equations, that there is a possible raise of discontinuities in solutions, even in the case when the initial conditions are smooth, see [19, page 390]. Here the concept of the classical solution is too restrictive, and therefore we seek a weak solution of this problem. To distinguish physically admissible solutions from nonphysical ones, entropy condition must be introduced, see [19, page 396]. By the solution of the problem (1)-(5),(6),(7) we mean the weak entropy solution of this problem in $Q_{\infty}$. The analysis to the solution of this problem can be found in many books, i.e. [4], [19], [5]. The general theorem on the solvability of the Riemann problem can be found in [4, page 88].

The solution is piecewise smooth and its general form can be seen in Fig. 1, where the system of half lines is drawn.

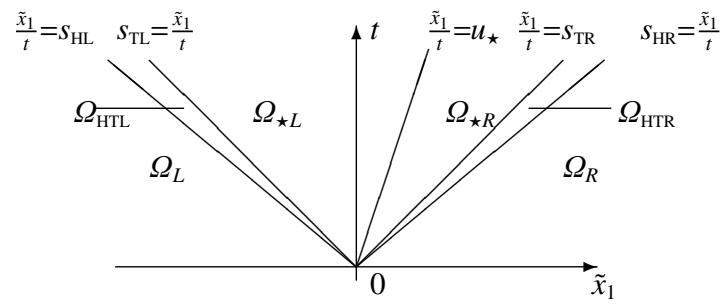

Fig. 1: Structure of the solution of the Riemann problem (1)-(5) ,(6),(7)

These half lines define regions, where solution is either constant or given by a smooth function. Let us define the open sets called wedges (see Fig. 1):

$$
\begin{aligned}
& \Omega_{L}=\operatorname{wedge}\left(L_{-\infty}, s_{\mathrm{HL}}\right), \\
& \Omega_{\star R}=\operatorname{wedge}\left(u_{\star}, s_{\mathrm{TR}}\right), \\
& \Omega_{\mathrm{HTL}}=\operatorname{wedge}\left(s_{\mathrm{HL}}, s_{\mathrm{TL}}\right) \text {, } \\
& \Omega_{\mathrm{HTR}}=\operatorname{wedge}\left(s_{\mathrm{TR}}, s_{\mathrm{HR}}\right) \text {, } \\
& \Omega_{\star L}=\operatorname{wedge}\left(s_{\mathrm{TL}}, u_{*}\right) \text {, } \\
& \Omega_{R}=\operatorname{wedge}\left(s_{\mathrm{HR}}, L_{+\infty}\right) \text {. }
\end{aligned}
$$

Using the theory in [4], [19], [5], we can write the solution for the primitive variables as

$$
\begin{aligned}
& \left.(\varrho, u, v, w, p)\right|_{\Omega_{L}}=\left(\varrho_{L}, u_{L}, v_{L}, w_{L}, p_{L}\right), \\
& \left.(\varrho, u, v, w, p)\right|_{\Omega_{\star L}}=\left(\varrho_{\star L}, u_{\star}, v_{L}, w_{L}, p_{\star}\right), \\
& \left.(\varrho, u, v, w, p)\right|_{\Omega_{\star R}}=\left(\varrho{ }_{\star}, u_{\star}, v_{R}, w_{R}, p_{\star}\right), \\
& \left.(\varrho, u, v, w, p)\right|_{\Omega_{R}}=\left(\varrho_{R}, u_{R}, v_{R}, w_{R}, p_{R}\right) .
\end{aligned}
$$

The folowing relations for these variables hold:

$$
\begin{aligned}
& u_{\star}=u_{L}+\left\{\begin{array}{l}
-\left(p_{\star}-p_{L}\right)\left(\frac{\frac{2}{(\gamma+1) \rho_{L}}}{p_{\star}+\frac{\gamma-1}{\gamma+1} p_{L}}\right)^{\frac{1}{2}}, p_{\star}>p_{L} \\
\frac{2}{\gamma-1} a_{L}\left[1-\left(\frac{p_{\star}}{p_{L}}\right)^{(\gamma-1) / 2 \gamma}\right], p_{\star} \leq p_{L}
\end{array}\right. \\
& \varrho_{\star L}=\left\{\begin{array}{l}
\varrho_{L} \frac{\frac{\gamma-1}{\gamma+1} \frac{p_{L}}{p_{\star}}+1}{\frac{p_{L}}{p_{\star}}+\frac{\gamma-1}{\gamma+1}}, p_{\star}>p_{L} \\
\varrho_{L}\left(\frac{p_{\star}}{p_{L}}\right)^{\frac{1}{\gamma}}, \quad p_{\star} \leq p_{L}
\end{array}\right. \\
& s_{T L}^{1}= \begin{cases}u_{L}-a_{L} \sqrt{\frac{\gamma+1}{2 \gamma} \frac{p_{\star}}{p_{L}}+\frac{\gamma-1}{2 \gamma}}, p_{\star}>p_{L} \\
u_{\star}-a_{L}\left(\frac{p_{\star}}{p_{L}}\right)^{(\gamma-1) / 2 \gamma}, \quad p_{\star} \leq p_{L}\end{cases} \\
& u_{\star}=u_{R}+\left\{\begin{array}{l}
\left(p_{\star}-p_{R}\right)\left(\frac{\frac{2}{(\gamma+1) g_{R}}}{p_{\star}+\frac{\gamma-1}{\gamma+1} p_{R}}\right)^{\frac{1}{2}}, p_{\star}>p_{R}, \\
-\frac{2}{\gamma-1} a_{R}\left[1-\left(\frac{p_{\star}}{p_{R}}\right)^{(\gamma-1) / 2 \gamma}\right], p_{\star} \leq p_{R}
\end{array}\right. \\
& \varrho_{\star R}=\left\{\begin{array}{l}
\varrho_{R} \frac{\frac{p_{\star}}{p_{R}}+\frac{\gamma-1}{\gamma+1}}{\frac{\gamma-1}{\gamma+1} \frac{p_{\star}}{p_{R}}+1}, p_{\star}>p_{R} \\
\varrho_{R}\left(\frac{p_{\star}}{p_{R}}\right)^{\frac{1}{\gamma}}, \quad p_{\star} \leq p_{R}
\end{array}\right. \\
& s_{T R}^{3}= \begin{cases}u_{R}+a_{R} \sqrt{\frac{\gamma+1}{2 \gamma} \frac{p_{\star}}{p_{R}}+\frac{\gamma-1}{2 \gamma}}, p_{\star}>p_{R} \\
u_{\star}+a_{R}\left(\frac{p_{\star}}{p_{R}}\right)^{(\gamma-1) / 2 \gamma}, \quad p_{\star} \leq p_{R}\end{cases}
\end{aligned}
$$

Here $a_{L}=\sqrt{\gamma p_{L} / \varrho_{L}}, a_{R}=\sqrt{\gamma p_{R} / \varrho_{R}}$, and $\gamma$ denotes the adiabatic constant. Further $s_{T L}^{1}$ denotes "unknown left wave speed", $s_{T R}^{3}$ "unknown right wave speed". Note, that the system (8) - (13) is the system of 6 equations for 6 unknowns $p_{\star}, u_{\star}, \varrho_{\star L} \varrho_{\star R}, s_{T L}^{1}, s_{T R}^{3}$.

Solution for the Pressure $p_{\star}$

The combination of the equations (8), (11) gives the implicit equation for the unknown pressure $p_{\star}$

$$
u_{L}+F_{1 L}\left(p_{\star}\right)=u_{R}+F_{3 R}\left(p_{\star}\right) \text {, }
$$

with

$$
F_{1 L}(p)=\left\{\begin{array}{ll}
-\left(p-p_{L}\right)\left(\frac{\frac{2}{(\gamma+1) Q_{L}}}{p+\frac{\gamma-1}{\gamma+1} p_{L}}\right)^{\frac{1}{2}}, & p>p_{L} \\
\frac{2}{\gamma-1} a_{L}\left[1-\left(\frac{p}{p_{L}}\right)^{(\gamma-1) / 2 \gamma}\right], & p \leq p_{L} .
\end{array},\right.
$$




$$
F_{3 R}(p)= \begin{cases}\left(p-p_{R}\right)\left(\frac{\frac{2}{(\gamma+)_{R}}}{p+\frac{\gamma-1}{\gamma+1} p_{R}}\right)^{\frac{1}{2}}, & p>p_{R}, \\ -\frac{2}{\gamma-1} a_{R}\left[1-\left(\frac{p}{p_{R}}\right)^{(\gamma-1) / 2 \gamma}\right], & p \leq p_{R} .\end{cases}
$$

This is a nonlinear algebraic equation, and one cannot express the analytical solution of this problem in a closed form. The solution $p_{\star}$ can be found as the root of the function $F(p)$ defined as

$$
F(p)=F_{3 R}(p)-F_{1 L}(p)+u_{R}-u_{L} .
$$

The analysis of this function can be found in [4]. Here we state, that $F(p)$ is monotone and concave. Also the analytic expression for its derivative is available. For a positive solution for the pressure $F(0)<0$ is required. This gives the pressure positivity condition

$$
u_{R}-u_{L}<\frac{2}{\gamma-1}\left(a_{L}+a_{R}\right)
$$

The Newton method can be used to find the root of $F(p)=$ 0 . With the pressure $p_{\star}$ known, we use the equations (8)(13) to obtain the whole solution.

\section{Remarks}

- Once the pressure $p_{\star}$ is known, the solution on the lefthand side of the contact discontinuity depends only on the left-hand side initial condition (6). And similarily, with $p_{\star}$ known, only the right-hand side initial condition (7) is used to compute the solution on the righthand side of the contact discontinuity.

- The solution in $\Omega_{L} \cup \Omega_{H T L} \cup \Omega_{\star L}$ (across 1 wave) (solvability in general case)

The solution components in $\Omega_{L} \cup \Omega_{H T L} \cup \Omega_{\star L}$ region must satisfy the system of equations (8)-(10). It is a system of three equations for four unknowns $\left(\varrho_{\star L}, p_{\star}\right.$, $\left.u_{\star}, s_{T L}^{1}\right)$. We have to add another equation in order to get the uniquely solvable system in $\Omega_{L} \cup \Omega_{H T L} \cup \Omega_{\star L}$. This is the key problem for the outlet boundary condition.

- The equation (8) can be written as the equation for pressure, see [7]

$$
\begin{gathered}
p_{\star}=P\left(u_{\star}\right), \\
P(u)=\left\{\begin{array}{l}
P_{S}(u), u<u_{L}, \\
P_{R}(u), u_{L} \leq u<u_{L}+\frac{2}{\gamma-1} a_{L},
\end{array}\right.
\end{gathered}
$$

with

$$
\begin{aligned}
P_{S}(u)= & p_{L}+\frac{\gamma+1}{4} \varrho_{L}\left(u_{L}-u\right)^{2}+ \\
& \frac{\left(u_{L}-u\right) \sqrt{4 \varrho_{L} \gamma p_{L}+\varrho_{L}^{2}\left(\frac{\gamma+1}{2}\right)^{2}\left(u_{L}-u\right)^{2}}}{2}, \\
& P_{R}(u)=p_{L}\left(\frac{-u+u_{L}+\frac{2}{\gamma-1} a_{L}}{\frac{2}{\gamma-1} a_{L}}\right)^{\frac{2 \gamma}{\gamma-1}} .
\end{aligned}
$$

In our work we use the analysis of this problem also for the solution of the initial-boundary value at the boundary faces.

\section{Boundary condition by preference of total quantities and the tangential component of velocity}

Using this boundary condition, we try to prescribe given total quantities at the face, comlete analysis was shown in [7], [20]. The conservation laws must be satisfied in close vicnity to the boundary face. We use the analysis of the incomplete Riemann problem to construct the values for the density, pressure and velocity. The following notation is used (some values are used only for an INLET case)
$\varrho_{B} \quad$ static density at the boundary (unknown)
$p_{B} \quad$ static pressure at the boundary (unknown)
$\boldsymbol{v}_{B} \quad$ velocity vector at the boundary (in global coordi- nates) (unknown)
$u_{B} \quad$ normal component of velocity at the boundary (lo- cal coordinates) (unknown)
$\boldsymbol{n}$ unit outer normal to face $\boldsymbol{n}=\left(n_{1}, n_{2}, n_{3}\right)$
$\varrho_{L} \quad$ static density near the wall, time 0
$p_{L} \quad$ static pressure near the wall, time 0
$\boldsymbol{v}_{L} \quad$ velocity vector, state near the wall at time 0
$u_{L} \quad$ normal component of velocity, state near the wall at time $0, u_{L}=\boldsymbol{v}_{L} \cdot \boldsymbol{n}$
$p_{o} \quad$ given value for the total pressure (=desired total pressure at the face)
$\theta_{o} \quad$ given value for the total temperature (=desired to- tal temperature at the face)
$\boldsymbol{d}$ given INLET velocity direction $\boldsymbol{d}=\left(d_{1}, d_{2}, d_{3}\right)$

At the vicinity of the boundary we solve the modified Riemann problem for the split Euler equations (1)-(5), introduced in Section 3, with the left-hand side initial condition (6) and the complementary conditions (prescribed direction of velocity and given total quantities).

$$
\begin{gathered}
\boldsymbol{v}_{B}=\boldsymbol{d}\left|\boldsymbol{v}_{B}\right|, \text { with } \boldsymbol{v}_{B} \cdot \boldsymbol{n}=u_{\star} . \\
\theta_{\star R}=\theta_{o}\left(1-\frac{\gamma-1}{2 a_{o}^{2}} u_{\star}^{2}\right), \text { with } a_{0}^{2}=\gamma R \theta_{0}(\boldsymbol{d} \cdot \boldsymbol{n})^{2}, \\
p_{\star}=p_{o}\left(\frac{\theta_{\star R}}{\theta_{0}}\right)^{\gamma /(\gamma-1)} \\
u_{\star}<0 .
\end{gathered}
$$

Here $\theta_{o}>0, p_{o}>0$ are given constants, $R$ denotes the characteristic gas constant, and $\gamma$ is the adiabatic constant. The equations (18),(19) are considered for the velocity $u_{\star} \in$ $\left(-\sqrt{\frac{2 a_{o}^{2}}{(\gamma-1)}}, \sqrt{\frac{2 a_{o}^{2}}{(\gamma-1)}}\right)$. The equations (18),(19) come from the idea, that the total pressure $p_{o}$ and the total temperature $\theta_{o}$ are known (prescribed) in the region $\Omega_{\star R}$.

According to analysis shown in [20], there are two possibillities for the 1-wave, either there is a 1-shock wave and the sought velocity $u_{\star}$ can be found as a root of the function

$$
F(u)=p_{o}\left(1-\frac{\gamma-1}{2 a_{o}^{2}} u^{2}\right)^{\gamma /(\gamma-1)}-P_{S}(u),
$$

considered for $u<u_{L},-\sqrt{\frac{2 a_{o}^{2}}{(\gamma-1)}}<u<0$, and with $P_{S}(u)$ defined in (16). 
Or there is a 1-rarefaction wave, and the velocity $u_{\star}$ can be computed combining (18),(19),(16) as

$$
\begin{gathered}
u_{\star}=\frac{2\left(u_{L}+\frac{2}{\gamma-1} a_{L}\right)-\sqrt{D I S}}{2\left[1+\frac{2 a_{L}^{2}}{(\gamma-1) a_{o}^{2}}\left(\frac{p_{o}}{p_{L}}\right)^{(\gamma-1) / \gamma}\right]}, \\
D I S=4\left(\frac{p_{o}}{p_{L}}\right)^{(\gamma-1) / \gamma} \frac{2 a_{L}^{2}}{(\gamma-1) a_{o}^{2}}\left[\frac{2 a_{o}^{2}}{(\gamma-1)}+\frac{4 a_{L}^{2}}{(\gamma-1)^{2}}\left(\frac{p_{o}}{p_{L}}\right)^{(\gamma-1) / \gamma}-\left(u_{L}+\frac{2 a_{L}}{\gamma-1}\right)^{2}\right] .
\end{gathered}
$$

Summarizing the above possibilities of the 1-shock and the 1-rarefaction wave we can construct the algorithm in figure 2 . for the computation of the velocity $u_{\star}$ in the star region.

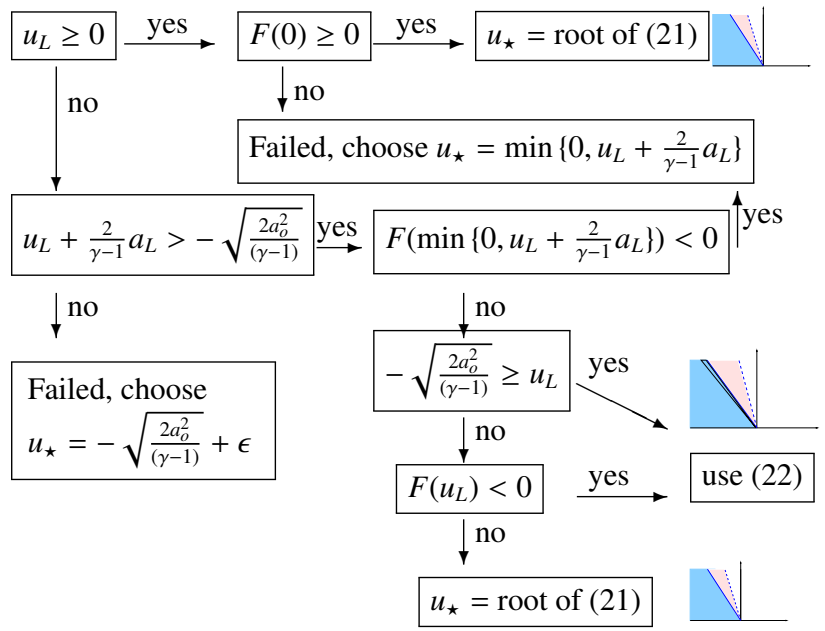

Fig. 2: Algorithm for the velocity solution $u_{\star}<0$.In the case of failure, the problem doesn't have a solution, and the values are chosen.

Knowing the velocity $u_{\star}$ we compute the pressure $p_{\star}$ using (19), and the density $\varrho_{\star R}=\frac{p_{\star}}{R \theta_{\star R}}$. At last we set the boundary values as

$$
p_{B}=p_{\star}, \varrho_{B}=\varrho_{\star R}, \quad \boldsymbol{v}_{B}=\frac{\boldsymbol{d} u_{\star}}{\boldsymbol{d} \cdot \boldsymbol{n}} .
$$

The problem (1)-(5),(6), (19), (18) has a unique solution for the initial data satisfying $u_{L}+\frac{2}{\gamma-1} a_{L}>-\sqrt{\frac{2 a_{o}^{2}}{(\gamma-1)}}$, and $F\left(\min \left\{0, u_{L}+\frac{2}{\gamma-1} a_{L}\right\}\right)>0$. In the case of $u_{L}+\frac{2}{\gamma-1} a_{L} \leq$ $-\sqrt{\frac{2 a_{o}^{2}}{(\gamma-1)}}$ there is no solution. In this case we prescribe the velocity $u_{\star}=-\sqrt{\frac{2 a_{o}^{2}}{(\gamma-1)}}+\epsilon$, with $\epsilon>0$ being a small positive constant. If $F\left(\min \left\{0, u_{L}+\frac{2}{\gamma-1} a_{L}\right\}\right) \leq 0$ then the problem does not have a negative solution, and for the practical applications we choose the velocity $u_{\star}=\min \left\{0, u_{L}+\frac{2}{\gamma-1} a_{L}\right\}$, or we use the boundary condition preferring the static pressure $p_{\star}=p_{o}$, see $[10,7]$, in this case.

Let $\tilde{p_{0}}, \tilde{\theta_{0}}$ be the given total quantities together with the given tangential velocity components : $v_{t}$ and $w_{t}$ are the velocity components in the direction of $\boldsymbol{o}$ and $\boldsymbol{p}$. Here $\boldsymbol{o} \cdot \boldsymbol{n}=$ $0,|\boldsymbol{o}|=1$, and $\boldsymbol{p}=\boldsymbol{n} \times \boldsymbol{o}$. Then we set

$$
\boldsymbol{d}=-\boldsymbol{n}, \quad \theta_{0}=\tilde{\theta_{o}}-\frac{\gamma-1}{2 \gamma R}\left(v_{t}^{2}+w_{t}^{2}\right), \quad p_{0}=\tilde{p_{0}}\left(\frac{\theta_{0}}{\tilde{\theta_{0}}}\right)^{\frac{\gamma}{\gamma-1}},
$$

and we use the same algorithm (figure 2.) for the computation of the velocity $u_{\star}$. Then it is $p_{B}=p_{\star}, \varrho_{B}=\varrho_{\star R}$, and

$\boldsymbol{v}_{B}=\left(n_{1} u_{\star}+o_{1} v_{t}+p_{1} w_{t}, n_{2} u_{\star}+o_{2} v_{t}+p_{2} w_{t}, n_{3} u_{\star}+o_{3} v_{t}+p_{3} w_{t},\right)$

\section{Outlet boundary condition by preference of massflow}

Using this boundary condition, we try to prescribe given total quantities at the face, comlete analysis was shown in $[8,14]$. The conservation laws must be satisfied in close vicnity to the boundary face. We use the analysis of the incomplete Riemann problem to construct the values for the density, pressure and velocity. The following notation is used (some values are used only for an INLET case)

$\varrho_{B} \quad$ static density at the boundary (unknown)

$p_{B} \quad$ static pressure at the boundary (unknown)

$\boldsymbol{v}_{B} \quad$ velocity vector at the boundary (in global coordinates) (unknown)

$u_{B} \quad$ normal component of velocity at the boundary (local coordinates) (unknown)

$\boldsymbol{n}$ unit outer normal to face $\boldsymbol{n}=\left(n_{1}, n_{2}, n_{3}\right)$

$\varrho_{L} \quad$ static density near the wall, time 0

$p_{L} \quad$ static pressure near the wall, time 0

$\boldsymbol{v}_{L} \quad$ velocity vector, state near the wall at time 0

$u_{L} \quad$ normal component of velocity, state near the wall at time $0, u_{L}=\boldsymbol{v}_{L} \cdot \boldsymbol{n}$

$G_{\star}$ given inlet massflow (=desired massflow at the face)

At the vicinity of the boundary we solve the modified Riemann problem for the split Euler equations (1)-(5), introduced in Section 3, with the left-hand side initial condition (6) and the complementary conditions prescribing the mass flow

$$
u_{\star} \varrho_{\star R}=G_{\star},
$$

where $G_{\star} \geq 0$ is given constant $\left(G_{\star}=\frac{\text { massflow }}{\text { face area }}\right)$. We are interested in the solution with $u_{\star}>0$, and $s_{L}$ ? $<0$, which guarantees the possibility of the values to be prescribed at the boundary. In general, there are two possibilities of the wave pattern, which may interest us.

\section{- 1-shock wave}

Here, we are interested in the solution with $s_{1}<0, u_{\star}>$ 0 . Let us construct the function $F_{S}(u)$, using the relations (8),(9)

$$
F_{S}(u)=u \varrho \star L(u)-G_{\star},
$$

where

$$
\varrho_{\star L}(u)=\varrho_{L} \frac{(\gamma-1) p_{L}+(\gamma+1) P_{S}(u)}{(\gamma+1) p_{L}+(\gamma-1) P_{S}(u)},
$$

and $P_{S}(u)$ defined in (16). The sought velocity $u_{\star}$ is the root of this function $F_{S}(u)$. It is $s_{1}<0$ for $u_{\star}<$ $u_{X}=u_{L} \frac{2+(\gamma-1) M_{L}^{2}}{(\gamma+1) M_{L}^{2}}$, with $M_{L}^{2}=u_{L}^{2} \varrho / \gamma p$, see [7]. The first derivative is $F_{S}^{\prime}>0$ for $u<\min \left\{u_{L}, u_{X}\right\}$. The problem has a solution with the 1 -shock wave if $F_{S}\left(\min \left\{u_{L}, u_{X}\right\}\right)>$ 0 . 


\section{- 1-rarefaction wave}

Let us assume the solution with the 1-rarefaction wave. Using (8),(9),(23), the velocity $u_{\star}$ is the root of the function

$$
F_{R}(u)=u \varrho_{L}\left[1-\frac{\gamma-1}{2 a_{L}}\left(u-u_{L}\right)\right]^{\frac{2}{\gamma-1}}-G_{\star},
$$

defined in the interval $u_{L}<u<u_{L}+\frac{2 a_{L}}{\gamma-1}$. It is $F_{R}\left(u_{L}\right)=$ $\varrho_{L} u_{L}-G_{\star}$. The first derivative is zero at the points $u_{1}=$ $u_{L}+\frac{2 a_{L}}{\gamma-1}, u_{0}=\frac{\gamma-1}{\gamma+1}\left(u_{L}+\frac{2 a_{L}}{\gamma-1}\right)$. Derivative $F_{R}^{\prime}(u)>0$ in the interval $\left(0, u_{0}\right)$ and $F^{\prime}(u)<0$ for $u \in\left(u_{0}, u_{1}\right)$. The maximum of the function $F_{R}$ is at the point $u_{0}$. We are interested in the solution with $s_{T L}<0$. This is satisfied only fo $u_{\star}<u_{0}$.

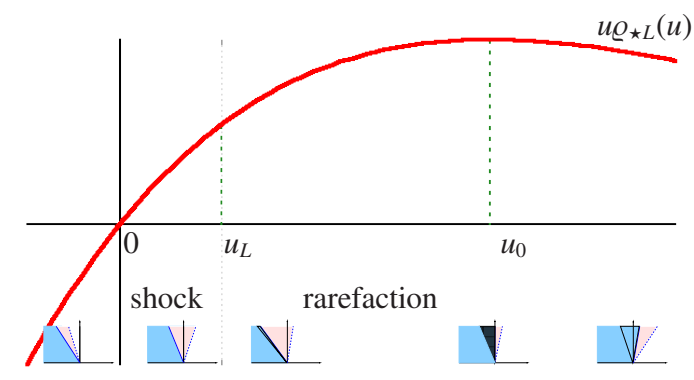

Fig. 3: Example of the function $u \varrho_{\star L}(u)$, here $u_{L}=20, \gamma=$ $1.4, \varrho_{L}=1.25, p_{L}=100000$.

\section{Algorithm}

Here we present the possible algorithm for the solution of the sought values at the boundary.

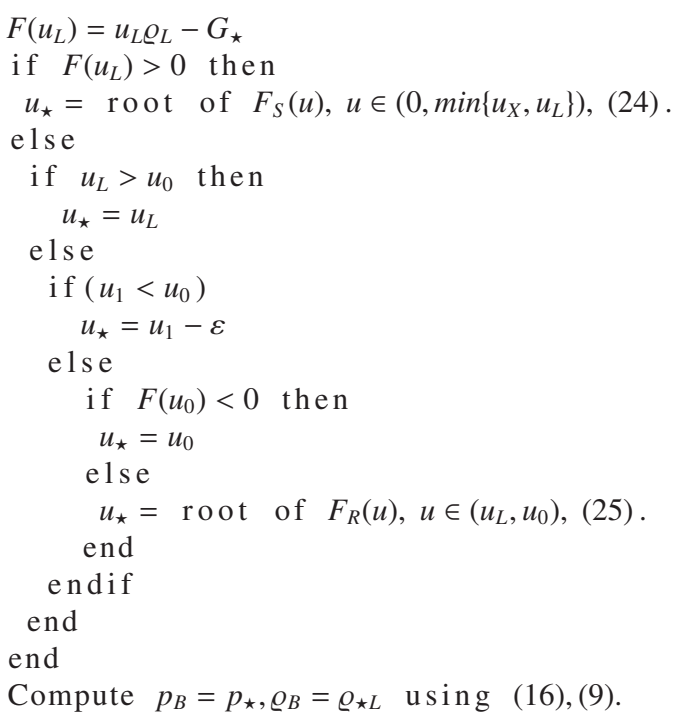

The resulting boundary velocity in the global coordinates is $\boldsymbol{v}_{B}=\boldsymbol{n}\left(u_{B}-u_{L}\right)+\boldsymbol{v}_{L}$.

\section{Simple fan simulation}

The simple fan simulation is based on the boundary conditions shown above in sections 4,5 . The inlet boundary condition with the given total temperature and the total pressure is connected with the outlet boundary condition
Table 1: Outlet boundary condition by the preference of massflow. The input data $u_{L}, G_{\star}$ and the solution (rounded) $\varrho_{B}$, $u_{B}, p_{B}, G_{B}=\varrho_{B} u_{B}$ for the test problems with $\varrho_{L}=1.25$, $p_{L}=100000$.

\begin{tabular}{|c||c||c|c|c|c|}
\hline \multicolumn{2}{|c|}{ input data } & \multicolumn{4}{c|}{ solution } \\
\hline$u_{L}$ & $G_{\star}$ & $G_{B}$ & $\varrho_{B}$ & $u_{B}$ & $p_{B}$ \\
\hline-20 & 1 & 1.00 & 1.1740 & 0.8517646 & 91596.49 \\
\hline-20 & 50 & 50.00 & 1.0113 & 49.44231 & 74326.85 \\
\hline-20 & 150 & 130.35 & 0.47304 & 275.5533 & 25655.28 \\
\hline 20 & 1 & 1.00 & 1.3235 & 0.7555568 & 108333.1 \\
\hline 20 & 100 & 100.00 & 0.96848 & 103.2548 & 69960.27 \\
\hline 20 & 200 & 150.45 & 0.53309 & 282.2200 & 30328.54 \\
\hline 400 & 10.0 & 10.00 & 3.2188 & 3.106711 & 421919.0 \\
\hline 400 & 100 & 100.00 & 3.0591 & 32.68887 & 385170.7 \\
\hline 400 & 600 & 500 & 1.25 & 400 & 100000 \\
\hline
\end{tabular}

with the preference of the mass flow. This procedure was succesfully used by authors in [3].

Here we suppose, that the total quantities $\theta_{0 F A N}, p_{0 F A N}$ and the tangential velocity components at the fan outlet are known. Then we may use the boundary condition in section 4 to compute the boundary state (at the fan outlet). The conservation law of mass is valid across the fan, and therefore we may use the known computed massflow (at the fan outlet) to complement the incomplete the Riemann problem at the fan inlet. The solution to this problem gives us the boundary state at the fan inlet, analysis and the algorithm is shown in section 5 .

The approach is based on the estimation of the total quantities $\theta_{0 F A N}, p_{0 F A N}$ and the tangential velocity at the fan, wich may be rather complicated. Let us for example suppose, that the distribution of the velocity $\boldsymbol{v}_{F A N}$ is known at the fan outlet (at some time instant), together with the total quantities $p_{0 X}, \theta_{0 X}$ before the fan rotor. Then we may estimate

$$
\begin{gathered}
\theta_{0 F A N}=\theta_{0 X}\left(1+\frac{\gamma-1}{2 \gamma R \theta_{0 X}} \boldsymbol{v}_{F A N}^{2}\right), \\
p_{0 F A N}=p_{0 X}\left(1+\frac{\gamma-1}{2 \gamma R p_{0 X}} \boldsymbol{v}_{F A N}^{2}\right)^{\gamma /(\gamma-1)} .
\end{gathered}
$$

The figures 4-5 show the test simulation of the fan $\left|\boldsymbol{v}_{F A N}\right|=$ $20\left[\mathrm{~m} \mathrm{~s}^{-1}\right]$ with the regime $p_{0 X}=101325[\mathrm{~Pa}], p_{0 X}=$ $293.15[\mathrm{~K}]$.

\section{Examples}

Here we show the computational results for the flow around and inside the high hall in the isothermal atmosphere. The initial condition was constant in the whole domain with the total temperature $\theta_{0}=293.15[\mathrm{~K}]$, zero velocity, and the total pressure distribution modified in the gravitational field as $p_{0}=p_{00} e^{9.81 z /\left(R \theta_{0}\right)}$, where $p_{00}=101325[\mathrm{~Pa}], z$ denotes the vertical coordinate, and $R$ is the gas constant $R=287.04\left[\mathrm{~J} \mathrm{~kg}^{-1} \mathrm{~K}^{-1}\right]$. The fan is placed horizontally in the high hall, both direction of the flow through the fan were simulated. The magnitude of the fan velocity (parameter) $\left|\boldsymbol{v}_{F A N}\right|=5\left[\mathrm{~m} \mathrm{~s}^{-1}\right]$ was chosen. Another simulations with two fans were computed. Results are shown in figures 6-8. 


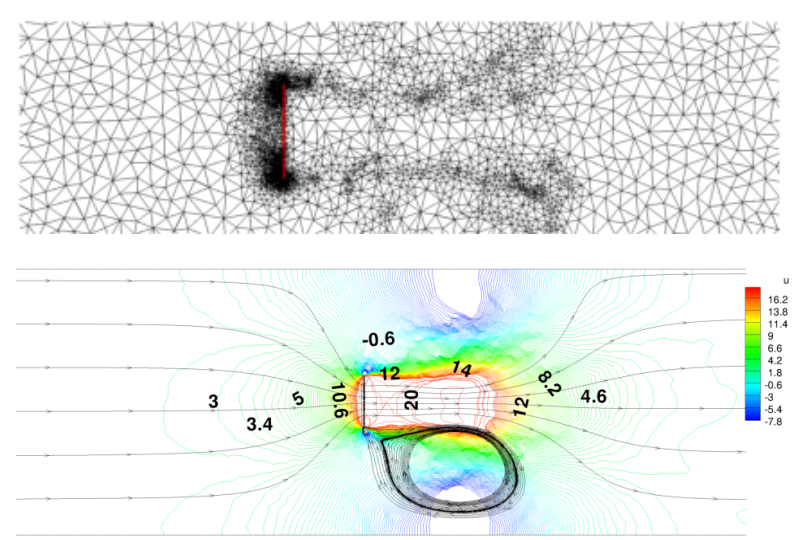

Fig. 4: The fan flow simulation example (fan depicted by the red vertical line), inviscid flow. Isolines of horizontal velocity, streamlines at time $t=0.15[\mathrm{~s}]$.

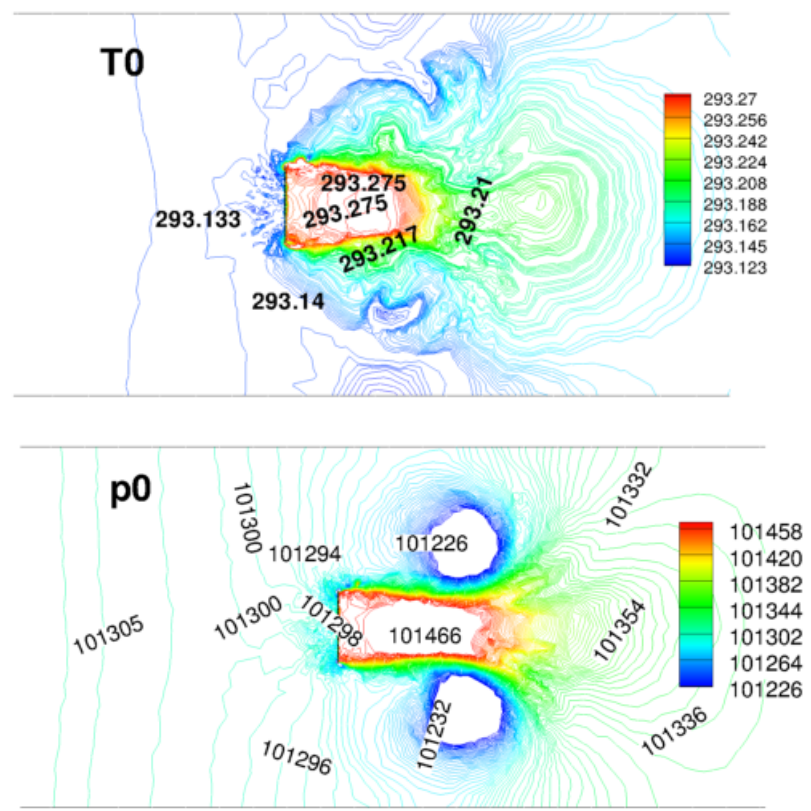

Fig. 5: The fan flow simulation example (fan depicted by the red vertical line), inviscid flow. Isolines of the total temperature and the total pressure, streamlines at time $t=0.15[\mathrm{~s}]$.

\section{Conclusion}

This paper shows the numerical simulation of the fan situated inside the high hall. The partial differential equations describing the conservation laws are solved numerically with the use of the finite volume method. Own software was programmed. The special modification of the Riemann problem and its solution was used at the boundaries. The combination of these boundary conditions was used for the simulation of the fan, which is the original result of this work.
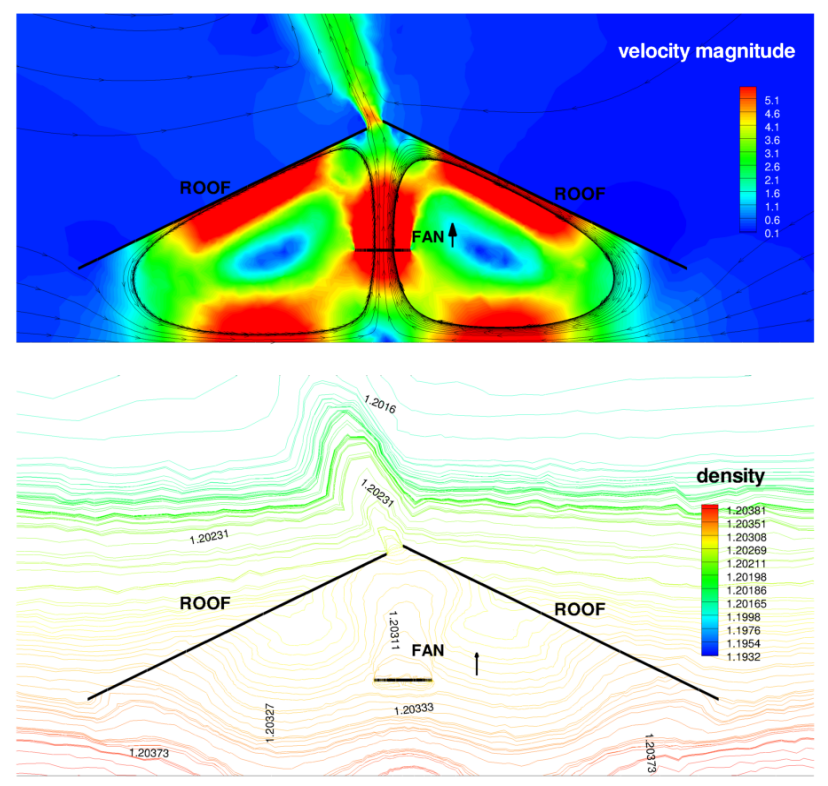

Fig. 6: The flow inside the high hall induced by the fan. One horizontally placed fan, facing upward. Density and velocity magnitude isolines and streamtraces shown.

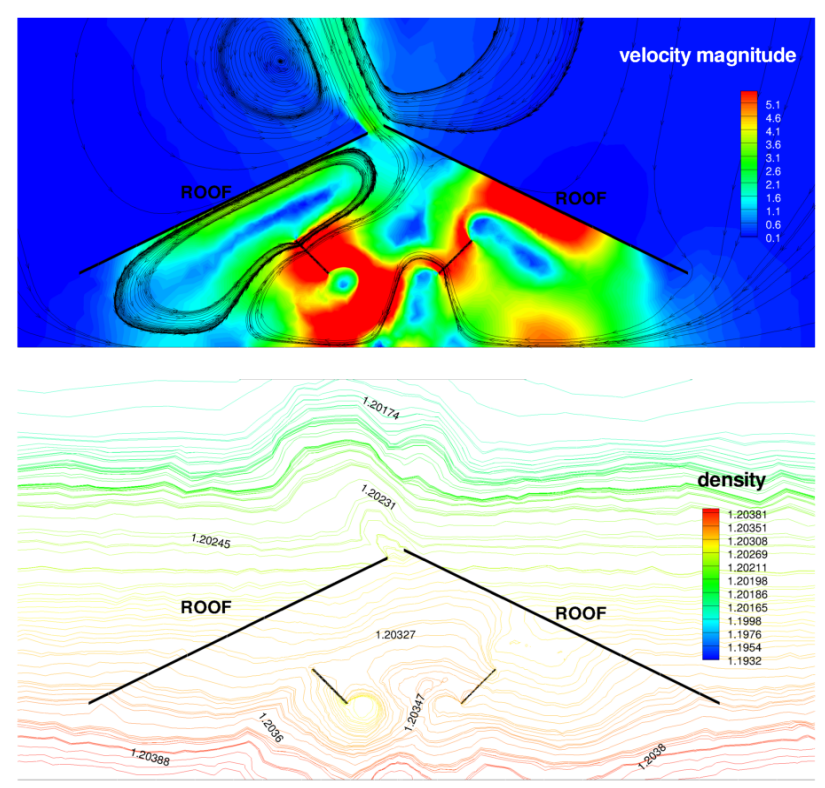

Fig. 7: The flow inside the high hall induced by two fans. Density and velocity magnitude isolines and streamtraces shown.

\section{Acknowledgment}

The results originated with the support of Ministry of the Interior of the Czech Republic, project SCENT, Grant MSM 0001066902 of the Ministry of Education of the Czech Republic, and Ministry of Industry and Trade of the Czech Republic for the long-term strategic development of the research organization. The authors acknowledge this support. 

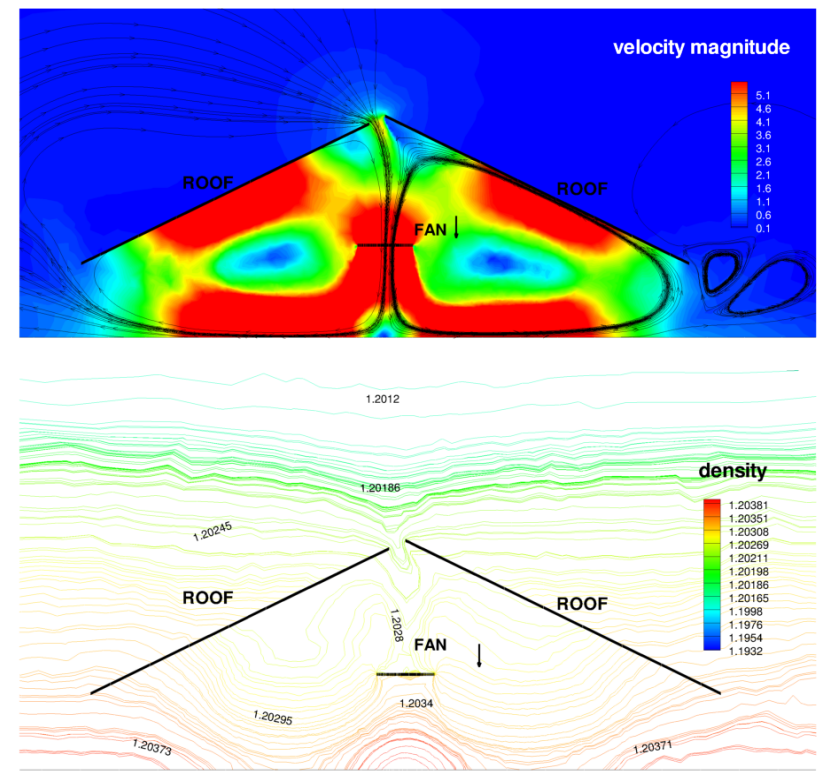

Fig. 8: The flow inside the high hall induced by the horizontally placed fan, facing downwards. Density and velocity magnitude isolines and streamtraces shown.

\section{References}

1. D. C. Wilcox, Turbulence Modeling for CFD. KNI, Inc., Anaheim, California, (July 1998).

2. C. J. Kok, AIAA Journal, Vol. 38., No. 7., (2000).

3. M. Kyncl and J. Pelant, EPJ WoC, 67, 02064 (2014)

4. M. Feistauer, J. Felcman, and I. Straškraba, Mathematical and Computational Methods for Compressible Flow, Oxford University Press, Oxford (2003)

5. E. F. Toro, Riemann Solvers and Numerical Methods for Fluid Dynamics, Springer, Berlin, (1997)

6. J. Pelant, ARTI Reports VZLÚ, Z-65, Z-67 to Z73,VZLÚ, Prague (1996-2000)

7. M. Kyncl, Numerical solution of the three-dimensional compressible flow. Doctoral Thesis, Prague (2011)

8. M. Kyncl and J. Pelant, Appl. Mech. and Mat., 821, 70$78,(2016)$

9. M. Kyncl and J. Pelant, EPJ WoC, 143, 02061 (2017)

10. M. Kyncl and J. Pelant, Applications of the NavierStokes equations for $3 d$ viscous laminar flow for symmetric inlet and outlet parts of turbine engines with the use of various boundary conditions, Tech. rep. R3998, VZLÚ (2006)

11. J. Michálek, P. Straka, Journal of Thermal Science, 22 (5), pp. 413-423, (2013)

12. P. Straka, Proc. ECCOMAS 2016, 5.-10.6.2016, pp. 7532-7543, Greece (2016).

13. P. Straka, The European Physical Journal of Conferences, 114, 02115 (2016)

14. M. Kyncl and J. Pelant, Proc. ECCOMAS 2016, 5.10.6.2016, Crete Island, Greece (2016).

15. M. Kyncl and J. Pelant, Implicit method for the $3 d$ RANS equations with the $k-\omega$ (Kok) Turbulent Model. Tech. rep. R5453, Prague (2012)

16. M. Kyncl and J. Pelant, Implicit method for the $3 D$ Euler equations. Tech. rep. R5375, VZLÚ (2012)

17. M. Kyncl and J. Pelant, EPJ WoC, 92, 02044 (2015)

18. M. Kyncl and J. Pelant, EPJ WoC, 45, 01097 (2016)
19. M. Feistauer, Mathematical Methods in Fluid Dynamics, Longman Scientific \& Technical, Harlow, (1993) 20. M. Kyncl and J. Pelant, Proc. ECCOMAS 2014, ECFD VI, 20.-25.7.2014, Barcelona, Spain (2014) 